\title{
Estimating Uterine Fibroid SNP-Based Heritability in European American Women with Imaging-Confirmed Fibroids
}

\author{
Michael J. Bray ${ }^{a, b} \quad$ Lea K. Davis ${ }^{b}$ Eric S. Torstenson ${ }^{b}$ Sarah H. Jones ${ }^{c}$ \\ Todd L. Edwards ${ }^{b-f}$ Digna R. Velez Edwards ${ }^{b-d, g, h}$
}

\begin{abstract}
${ }^{a}$ Department of Psychiatry, Washington University School of Medicine, St. Louis, MO, USA; b Vanderbilt Genetics Institute, Vanderbilt University Medical Center, Nashville, TN, USA; ' Institute for Medicine and Public Health, Vanderbilt University Medical Center, Nashville, TN, USA; ${ }^{d}$ Vanderbilt Epidemiology Center, Vanderbilt University Medical Center, Nashville, TN, USA; ' Department of Medicine, Vanderbilt University Medical Center, Nashville, TN,

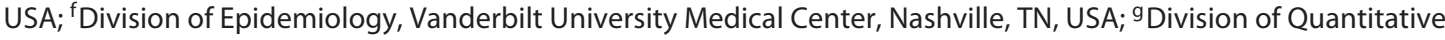
Sciences, Department of Obstetrics and Gynecology, Vanderbilt University Medical Center, Nashville, TN, USA; h Department of Biomedical Informatics, Vanderbilt University Medical Center, Nashville, TN, USA
\end{abstract}

\section{Keywords}

Leiomyomata $\cdot$ Heritability · Genome-wide SNP data Uterus · Ultrasound

\begin{abstract}
Background: Heritability estimates (including twin and single nucleotide polymorphism [SNP]-based heritability studies) for fibroids have been inconsistent across prior studies ranging between 9 and $69 \%$. These inconsistencies are due to variations in study design and included populations. A major design issue has been lack of imaging confirmation to identify controls, where asymptomatic women without imaging confirmation may be misclassified as controls leading to an attenuation of heritability estimates. To reconcile the differences in prior heritability estimates and the impact of misclassification of controls on heritability, we determined SNP-based heritability and characterized the genetic architecture of pelvic image-confirmed fibroid cases and controls. Methods: Analyses were performed among women of Euro-
\end{abstract}

\section{KARGER}

(c) 2019 S. Karger AG, Basel

E-Mail karger@karger.com

www.karger.com/hhe pean American descent using genome-wide SNP data from BioVU, a clinical database composed of DNA linked to deidentified electronic health records. We estimated the genetic variance explained by all SNPs using Genome-Wide Complex Trait Analysis on imputed data. Fibroid cases and controls were identified using a previously reported phenotyping algorithm that required pelvic imaging confirmation. Results: In total, we used 1,067 image-confirmed fibroid cases and 1,042 image-confirmed fibroid controls. The SNPbased heritability estimate for fibroid risk was $h^{2}=0.33 \pm$ $0.18(p=0.040)$. We investigated the relationship between heritability per chromosome and chromosome length $\left(r^{2}<\right.$ $1 \%)$, with chromosome 8 explaining the highest proportion of variance for fibroid risk. There was no enrichment for intergenic or genic SNPs for the fibroid SNP-based heritability. Excluding loci previously associated with fibroid risk from genome-wide association study did not attenuate fibroid heritability suggesting that loci associating with fibroid risk are yet to be discovered. Conclusions: We observed that fibroid SNP-based heritability was higher than the previous 
estimate using genome-wide SNP data that relied on selfreported outcomes, but within the range of prior twin pair studies. Furthermore, these data support that imprecise phenotyping can significantly affect the ability to estimate heritability using genotype data.

(c) 2019 S. Karger AG, Basel

\section{Introduction}

Uterine leiomyoma (fibroids) are benign tumors of the uterus [1], and up to $70 \%$ of women will develop a fibroid by menopause [2]. Symptoms of fibroids include abnormal bleeding during menstruation, increased urinary frequency, and pelvic pressure [3, 4]. Likely because of the symptoms, fibroids are the leading cause of hysterectomy in the US [5]. Annual US costs for fibroids is estimated to be between 5.9 and 34.4 billion USD [6].

Heritability estimates for fibroids have been inconsistent across prior studies. One twin study of European American (EA) women, limited to women who underwent hysterectomies with pelvic imaging used to determine fibroid status, estimated fibroid heritability to be $69 \%$ [7]. Another twin study randomly selected samples of monozygotic and dizygotic twins from the Finnish Twin Cohort study and evaluated ultrasound characteristics across twin pairs with and without fibroids; this study estimated the heritability for fibroid number to be $26 \%$ [8]. Finally, a study using genome-wide single nucleotide polymorphism (SNP) data estimated the SNP-based heritability of fibroids to be 9\% [9]. Although well powered $(n=57,151)$, this study had a low fibroid prevalence $(2.77 \%)$ and relied on self-report fibroid status [9]. It is possible that the inconsistencies regarding heritability estimates across these studies are due to how fibroids were classified and how subjects were recruited. For example, up to $51 \%$ of asymptomatic cases may be misclassified as controls if a study relied on self-report alone, thus selfreported studies result in phenotypic heterogeneity $[2,9]$.

There have been three large-scale genetic studies of fibroids that include two genome-wide association studies (GWAS) of fibroids and one genome-wide linkage scan [10-12]. The GWAS by Cha et al. [11] found three loci, $10 \mathrm{q} 24.33$ (rs7913069; $\left.p=8.65 \times 10^{-14}\right), 22 \mathrm{q} 13.1$ $\left(\mathrm{rs} 12484776 ; p=2.79 \times 10^{-12}\right)$, and $11 \mathrm{p} 15.5$ (rs2280543; $\left.p=3.82 \times 10^{-12}\right)$, associated with fibroid risk in a Japanese cohort. These loci were replicated in an EA population $[13,14]$. In another GWAS performed among African American women, a genome-wide significant association was observed between rs739187 in cytohesin 4 (CYTH4) in 22q13.1 and fibroid risk [12]. Finally, a linkage study identified two significant linkage peaks at 10p11 (logarithm of odds $=4.15)$ and $3 \mathrm{p} 21$ (logarithm of odds = 3.73). They also identified an SNP (CCDC57, rs4247357, $p=3.05 \times 10^{-8}$ ) associated with fibroids within the linkage regions.

Current GWAS of fibroids have identified only a few variants that explain a small proportion of fibroid risk, as expected based on the available sample sizes. Better characterization of fibroid heritability, with well-phenotyped populations, is needed to understand the genetic architecture influencing risk for fibroids. Our objective was to reconcile differences reported across heritability estimates from twin studies and those using GWAS data and to assess the role of phenotyping on heritability estimates by estimating and characterizing the SNP-based heritability of image-confirmed fibroids in EA women using GWAS data.

\section{Materials and Methods}

\section{Study Population}

Detailed descriptions of the biorepository at the Vanderbilt University Medical Center (BioVU) have been reported previously $[15,16]$. The BioVU (2007-present) consists of DNA linked to de-identified electronic health records. The electronic health records include clinical and demographic data for each patient at the Vanderbilt University Medical Center [15]. Studies using the BioVU are classified as non-human subjects research by the Institutional Review Boards and the Office of Human Research Protections [16].

Fibroid cases and controls were identified using a validated phenotyping algorithm with a positive predictive value of $96 \%$ and a negative predictive value of $98 \%$ [17]. The algorithm classified an individual as a case if the individual was listed as white, was at least 18 years old, and had International Classification of Diseases, ninth revision (ICD-9) or Current Procedural Terminology codes for at least one pelvic imaging or surgery for treating fibroids and for a fibroid diagnosis [17]. Controls were at least 18 years old, had at least two ICD-9 or Current Procedural Terminology codes for pelvic imaging, and had no history of myomectomy, hysterectomy, or uterine artery embolization [17] and were group-matched by ancestry to cases. We also conducted a case-only analysis to evaluate the SNP-based heritability of fibroid size. To determine the fibroid size, we manually abstracted fibroid measurements from imaging and surgical reports. Patient demographic information was recorded at the time of diagnosis.

Outcome measurements include fibroid presence (case vs. control), largest fibroid dimension of all measurements, and largest fibroid volume. Fibroid volume was calculated using the equation of an ellipsoid: length $\times$ width $\times$ height $\times 0.523$. For the outcome to have a normal distribution, the fibroid largest dimension and volume were $\log _{10}$ transformed. Approximately a third of individuals with volume measurements had their third fibroid dimension measurement imputed by taking the mean of the first two 
measurements. This study was evaluated and approved by the Vanderbilt University Medical Center Institutional Review Board. In addition, all methods were carried out in accordance with relevant regulations and guidelines.

\section{Genotyping}

Genotyping of BioVU EA individuals was performed on the Affymetrix Axiom Biobank array (Affymetrix, Inc., Santa Clara, CA, USA) using PicoGreen (Invitrogen, Inc., Grand Island, NY, USA) to purify and to quantify the DNA.

\section{Quality Control of Genome-Wide Genotype Data}

Genetic data had standard quality control (QC) prior to imputation using PLINK 1.07 software [18]. Pre-imputed QC included: removing subjects with low genotyping efficiency $(<95 \%)$, removing related individuals (both individuals from a pair with a probability of identity by descent [IBD] of $>0.95$ and one from a pair with a probability of IBD from 0.2 to 0.95 ), removing individuals with inconsistency between genetic and reported sex. Additionally, we removed SNPs out of Hardy-Weinberg equilibrium (HWE) $\left(p \leq 10^{-6}\right)$, SNPs with a low genotyping efficiency $(<95 \%)$, SNPs with a minor allele frequency (MAF) $<0.01$, and SNPs without a chromosome location. Finally, SNPs were aligned to the + strand of the 1,000 Genomes (build 37, 2013).

We imputed non-genotyped SNPs using IMPUTE2.3.0 software [19] and subsequently converted the imputed genetic to the PLINK 1.07 format [18] after filtering SNPs with an info score $\leq 0.95$ and removing insertion/deletion polymorphisms. We then applied the Genome-Wide Complex Trait Analysis (GCTA)-specific QC that was performed by Lee et al. [20], which included: removing SNPs with an MAF $<0.05$, removing related individuals (one from a pair with a probability of IBD $>0.05$, and removing SNPs out of HWE $(\geq 0.05)$. Post-QC SNP numbers included 4,500,362 for fibroid risk, 4,522,829 for fibroid volume, and 4,518,340 for largest fibroid dimension (Appendix Table A1). In addition, post-QC numbers of individuals for each outcome are shown in Appendix Table A1.

\section{Statistical Analyses}

Covariate and demographic data were summarized by Stata/SE (College Station, TX, USA). Principal components (PCs) were created using EIGENSTRAT4.2 software [21]. To reduce admixture within BioVU, we removed all samples whose first PC (PC1) and second PC (PC2) were more than four standard deviations from the mean of PC1 and PC2 of the European individuals from the 1,000 Genomes (Appendix Fig. A1 and A2).

We estimated the genetic variance explained by all SNPs using GCTA software [22]. GCTA creates a genetic relationship matrix (GRM) between pairs of individuals within a cohort using all SNPs. GCTA uses a mixed linear model that estimates the genetic variance explained by all SNPs for a trait using the restricted maximum likelihood approach [22]. The final model for fibroid risk included the GRM, which was modeled as a random effects term and the fixed effects terms age at diagnosis, body mass index (BMI), and top five PCs. We transformed the observed estimate of SNP-based heritability to the liability scale using a fibroids prevalence estimate of $70 \%$. A prevalence estimate of $70 \%$ was used in models on fibroid risk because the mean age of our cohort was 50.8 and previous cumulative incidence estimates were $\sim 70 \%$ for women of $\mathrm{Eu}$ ropean ancestry near 51 years of age [2]. SNP-based heritability
Table 1. Demographic characteristics of BioVU European American women

\begin{tabular}{ll}
\hline Age, years $(n=2,109)$ & $50.8 \pm 16$ \\
BMI $(n=2,109)$ & $28.6 \pm 7$ \\
Fibroid risk $(n=2,109)$ & \\
$\quad$ Control, $\%(n=1,042)$ & 59 \\
$\quad$ Case, $\%(n=1,067)$ & 51 \\
Fibroid volume, $\mathrm{cm}^{3}(n=373)$ & $9.6(2.3-39.0)$ \\
Largest fibroid dimension, cm $(n=551)$ & $2.6(1.6-4.4)$ \\
\hline
\end{tabular}

Figures are mean \pm SD or median with $\mathrm{IQR}$ in parentheses. BMI, body mass index; SD, standard deviation; IQR, interquartile range.

estimates for a range of prevalences are shown in Appendix Table A2. Secondary analyses included estimating SNP-based heritability of fibroid size, namely volume and largest dimension. Final models for fibroid volume and max dimension were adjusted for age at diagnosis, BMI, and five PCs without a prevalence adjustment. To show that potential residual population stratification did not lead to spurious SNP-based heritability estimates, we performed sensitivity analyses where SNP-based heritability estimates were calculated adjusting for age at diagnosis, BMI, and varying numbers of PCs.

To characterize the genetic architecture of fibroids, we partitioned the genetic variance into separate GRMs by chromosomes and by genic and intergenic regions, respectively. We annotated SNP location into genic/intergenic regions using ANNOVAR software [23]. We used a distance of $1 \mathrm{~kb}$ from a transcript as the boundary threshold for genic and intergenic SNPs. Additional subanalyses involved excluding genomic regions previously implicated in fibroid risk based on GWAS. Exclusion criteria included selecting SNPs from final meta-analyses of GWAS $[11,12]$ with a $p$ value $\leq 1.00 \times 10^{-6}$ and subsequently removing all loci $\pm 0.5 \mathrm{Mb}$ of these selected SNPs to account for linkage disequilibrium in the region. Lastly, there is a common inversion that spans approximately $12 \mathrm{cM}$ on chromosome 8 encompassing 8p23.1-8p22 with a frequency of approximately $21 \%$ in European populations [24]. We determined the effect that this inversion has on fibroid risk by removing it from chromosome-specific SNP-based heritability estimates and comparing the difference in point estimates.

\section{Results}

\section{Demographic Data}

There were 2,109 EA women (1,067 cases and 1,042 controls) included in these analyses (Table 1). Their mean age was $50.8 \pm 16$ years, and most women were overweight or obese (mean BMI: $28.6 \pm 7$ ). There were 373 cases with fibroid volume measurements (median of largest volume: $9.6 \mathrm{~cm}^{3}$ ) and 551 cases with fibroid dimension measurements (median of largest dimension: $2.6 \mathrm{~cm}$ ). 
Table 2. SNP-based heritability estimates for fibroid risk using European Americans within BioVU

\begin{tabular}{lll}
\hline Outcome & $\begin{array}{l}\text { SNP-based heritability } \\
\text { estimate } \pm \text { SE }\end{array}$ & $p$ value \\
\hline Fibroid risk & \\
Largest fibroid dimension & $0.35 \pm 0.47$ & $0.040^{\mathrm{b}}$ \\
Largest fibroid volume & $0.14 \pm 0.66$ & 0.238 \\
\end{tabular}

Model: adjusted for age, BMI, and 5 PCs. SNPs are limited to $\geq 5 \%$ MAF. ${ }^{\text {a }}$ Prevalence estimates of fibroid risk is set at $70 \%$. ${ }^{\mathrm{b}}$ Statistically significant.

Table 3. SNP-based heritability estimates from partitioned SNPs into intergenic and genic regions

\begin{tabular}{lll}
\hline Location & $\begin{array}{l}\text { SNP-based heritability } \\
\text { estimate } \pm \text { SE }\end{array}$ & Number of SNPs ${ }^{\mathrm{a}}$ \\
\hline Intergenic & $0.21 \pm 0.15$ & 2.80 \\
Genic & $0.12 \pm 0.14$ & 1.69
\end{tabular}

Model: adjusted for age, BMI, and 5 PCs. SNPs are limited to $\geq 5 \%$ MAF. Prevalence estimates of fibroids are set at $70 \%$. ${ }^{a}$ In $1,000,000$ SNPs.

\section{SNP-Based Heritability Estimation}

The SNP-based heritability estimate for fibroid risk, or the proportion of phenotypic variance that was explained by genetic variance, was $h^{2}=0.33 \pm 0.18(p=0.040)(\mathrm{Ta}-$ ble 2), and this estimate was robust to a MAF threshold of $1 \%$. The SNP-based heritability estimates for the fibroid largest dimension was $h^{2}=0.35 \pm 0.47(p=0.238)$, and for volume it was $h^{2}=0.14 \pm 0.66(p=0.417)$. These SNP-based heritability estimates for each outcome were similar after adjusting for a range of PCs (risk $h^{2}=0.29$ 0.38 ; largest dimension $h^{2}=0.34-0.44$; volume $h^{2}=0.12-$ 0.35) (Appendix Tables A3-A5).

The correlation $\left(r^{2}\right)$ between chromosome length in base pairs and SNP-based heritability per chromosome was $0.89 \%(p=0.669)$ (Fig. 1). Chromosome 8 explained the greatest proportion of variance for fibroid risk (8.76\%), followed by chromosome 10 (5.57\%), 11 (5.11\%), and 7 (2.65\%). The summation of the SNP-based heritability estimate of each chromosome was $34 \%$, which is not significantly different from the univariate estimate suggesting that residual population stratification and cryptic relatedness are well controlled [25]. In addition, there is an approximately $12-\mathrm{cM}$ common inversion

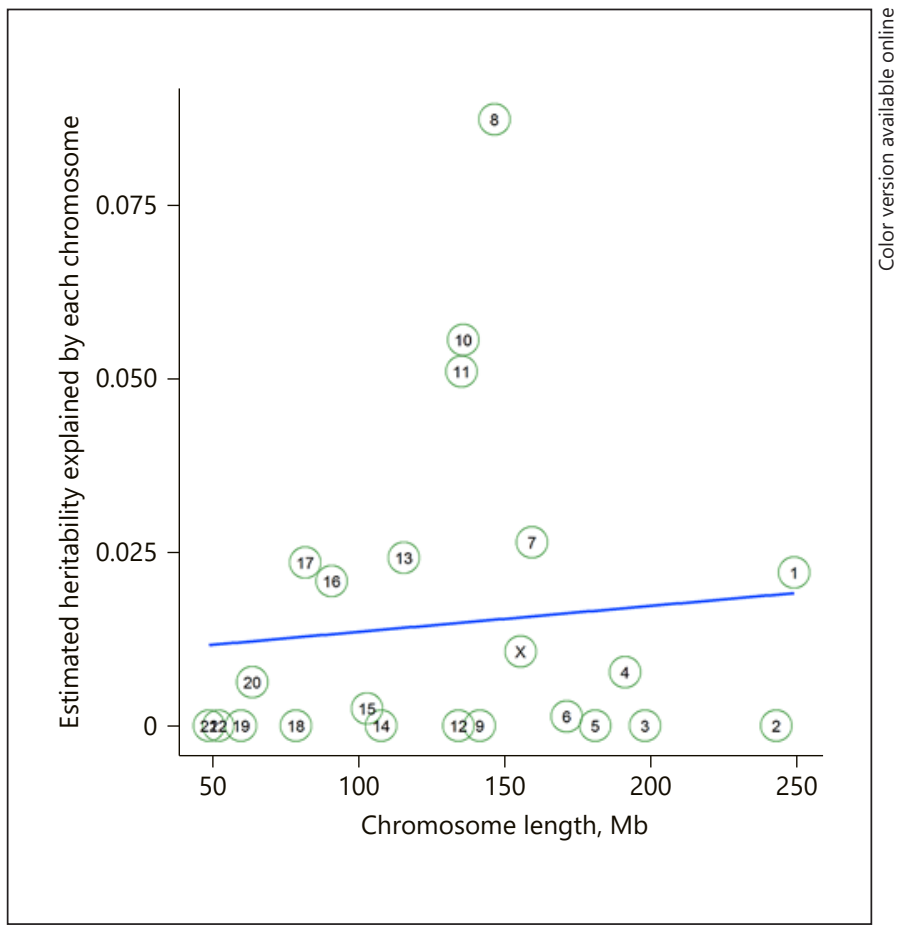

Fig. 1. Estimated SNP-based heritability of each chromosome by chromosome length. $R^{2}$ is $0.89 \%$, and the $p$ value of the model is 0.669 .

present at a frequency of $21 \%$ in EA populations on chromosome 8 spanning $8 \mathrm{p} 23.1-8 \mathrm{p} 22$ [24]. It is possible that this inversion could affect the disproportionate contribution of SNP-based heritability for chromosome 8 . Because of this, we estimated fibroid SNP-based heritability on chromosome 8 excluding $8 \mathrm{p} 23.1-8 \mathrm{p} 22,8 \mathrm{p}$, and $8 \mathrm{q}$, respectively (Appendix Figure A3). We found that excluding 8p23.1-8p22 did not change the contribution of SNP-based heritability from chromosome 8 . We also found that most of the SNP-based heritability from chromosome 8 was located in 8q. Even though fibroids are a sex-linked disease and have a hormonal component especially with estrogen [26], we did not observe enrichment of SNP-based heritability on chromosome X which contributed about $1 \%$ to the overall estimate (proportional to the number of X chromosome SNPs included in the analysis).

We also partitioned the genetic data into genic and intergenic regions resulting in 2,801,797 intergenic SNPs (62.3\%) and 1,694,891 genic SNPs (37.7\%) (Table 3). There was no enrichment for intergenic or genic SNPs for the SNP-based heritability of fibroid risk (Fig. 2). 
Fig. 2. Proportions of SNP-based heritability estimates and percentage of SNPs pie charts for SNPs that were partitioned into intergenic and genic SNPs.

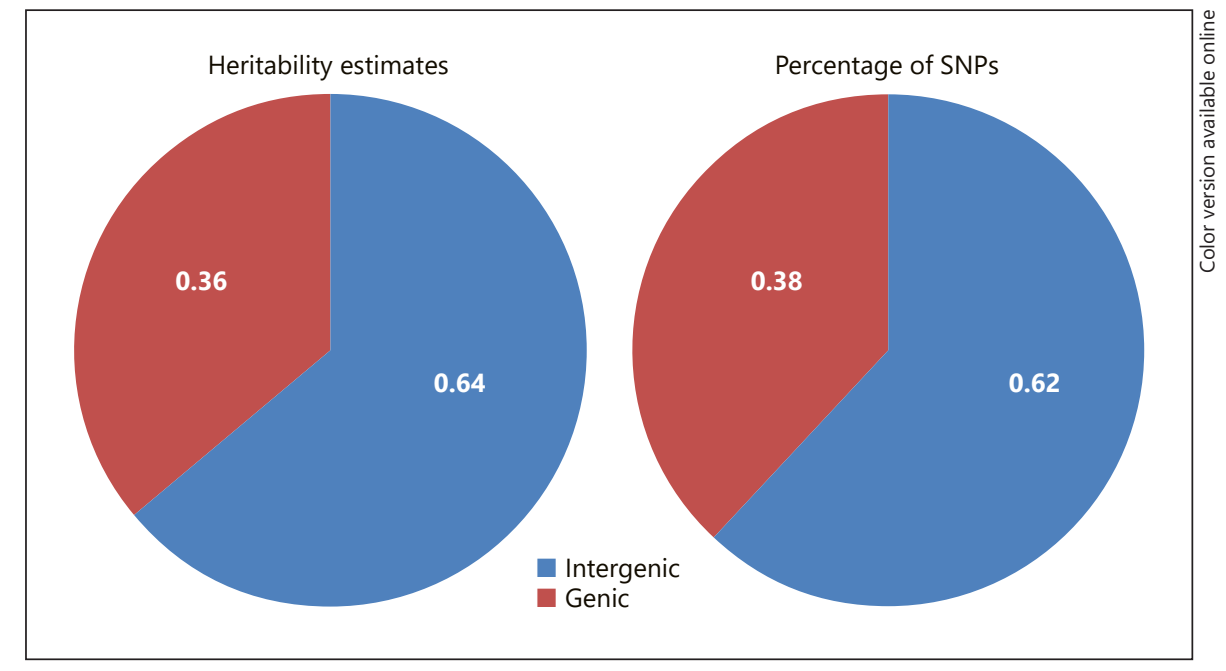

SNP-Based Heritability Explained by Known Fibroid GWAS Loci

The genomic regions that met the exclusion criteria were $10 \mathrm{q} 24.33-10 \mathrm{q} 25.1,11 \mathrm{p} 15.5$, and 22q13.1-22q13.2 from the GWAS by Cha et al. [11] and 3p26.1, 8p23.2, 8q21.11, and 22q12.3-22q13.1 from the GWAS by Hellwege et al. [12]. Excluding genetic loci previously associated with fibroid risk from all previous GWAS $[11,12]$ did not attenuate the estimated SNP-based heritability of fibroid risk ( $h^{2}$ from all studies $\left.=0.34\right)$ (Appendix Table A6).

\section{Discussion}

This was the first study to estimate and characterize the SNP-based heritability of fibroids using a dataset of EA women with image-confirmed fibroid status. Estimated SNP-based heritability of fibroid risk was $0.33 \pm 0.18$. Chromosome 8 contributed the greatest proportion of SNPbased heritability, and we observed little correlation between chromosome length and chromosomal SNP-based heritability. There was no enrichment of fibroid SNP-based heritability for intergenic or genic regions. Finally, censoring genetic loci previously implicated in fibroid risk did little to attenuate the estimated SNP-based heritability.

Our SNP-based heritability estimate for fibroid risk was within the range of estimates previously reported in twin studies (26-69\%) [7, 8]. The first study by Snieder et al. [7] conducted in a sample of 98 monozygotic and 125 dizygotic twin pairs from the UK found the heritability of fibroids to be $69 \%$. At least one twin from each pair underwent a hysterectomy. The high heritability from this study could be because they used hysterectomy as a criterion for selecting participants, which may result in overrepresentation of severe fibroids and thus greater genetic liability. The second study conducted ultrasounds on randomly selected twin pairs (17 monozygotic and 16 dizygotic) from the Finnish Twin Cohort. They estimated heritability of fibroid number at $26 \%$, closer to our fibroid risk heritability estimate [8].

Our SNP-based heritability estimate was higher than previous SNP-based heritability estimates from genomewide SNP data by Ge et al. [9]. This study had a sample size of 57,151 composed of women from the UK Biobank aged 40-69 years with self-reported outcomes [9] and a fibroid prevalence of only $2.77 \%$. When compared to the general population, this prevalence rate is low, suggesting potential misclassification in phenotype definition [9]. For example, a study by Baird et al. [2] observed the cumulative incidence of fibroids to be approximately $70 \%$ for white women aged 49 years in a study where women were systematically screened by ultrasounds. The large discrepancy between prevalence estimates between the two studies could signal potential misclassification for fibroid controls resulting in a lower SNP-based heritability estimate in their study.

While we estimated the SNP-based heritability for the fibroid largest dimension and volume, our same sizes were too small to get reliable estimates. Future studies with larger sample sizes are needed to evaluate the SNPbased heritability of fibroid size. In addition, while our phenotyping algorithm is improved with regard to outcome classification of cases and controls [17], our sample size was on the minimum threshold for performing such SNP-based heritability estimates. 
We present the first estimate of fibroid SNP-based heritability using a dataset of EA women with image-confirmed fibroid status using genotype data. We observed that fibroid SNP-based heritability in our study was higher than a previous genetic estimate using GWAS data which we believe is due to including only image-confirmed cases and controls in our analysis. In addition, we observed that our fibroid SNP-based heritability estimate was not significantly attenuated when censoring genetic loci previously associated with fibroid risk, suggesting that many of the genetic loci that are associated with fibroid risk in EA women are yet to be discovered. Larger future studies on fibroid risk are needed to understand the genetic underpinnings of fibroid SNP-based heritability in EA women.

\section{Statement of Ethics}

The authors have no ethical conflicts to disclose.

\section{Disclosure Statement}

The authors have no conflicts of interest to disclose.

Table A1

Final sample size and SNP count for each outcome

\begin{tabular}{lcl}
\hline Outcome & Sample size & $\begin{array}{l}\text { Post-QC SNP number - } \\
\text { MAF 5\% }{ }^{\mathrm{a}}\end{array}$ \\
\hline Fibroid risk & & $4,500,362$ \\
$\quad$ Case & 1,042 & \\
$\quad$ Control & 1,067 & \\
Volume & 373 & $4,522,829$ \\
Max dimension & 551 & $4,518,340$ \\
\hline
\end{tabular}

${ }^{a}$ Post-QC SNP numbers. These are slightly different between studies because we removed SNPs out of HWE at the end for each outcome independently.

\section{Funding Sources}

This study was funded by the National Institutes of Health (NIH)grants(R01HD074711, R03HD078567, andR01HD093671) to Digna R. Velez Edwards and by the Human Genetic Training Grant (5T32GM080178) and the VICTR Training Grant (6TL1TR000447) to Michael J. Bray.

The publication described was supported by CTSA award No. UL1TR000445 from the National Center for Advancing Translational Sciences. Its contents are solely the responsibility of the authors and do not necessarily represent official views of the National Center for Advancing Translational Sciences or the National Institutes of Health.

\section{Author Contributions}

M.J.B.: Designed the study, conducted analyses, drafted the manuscript.

L.K.D.: Designed the study and edited the manuscript.

E.S.T.: Conducted the analysis of data and edited the manuscript.

S.H.J.: Conducted the data quality control, data preparation, and edited the manuscript.

T.L.E.: Designed the study, conducted analyses, contributed to the first manuscript draft and edited the manuscript.

D.R.V.E.: Designed the study, contributed to the first manuscript draft and edited the manuscript.

\section{Table A2}

Heritability estimates for fibroid risk using European Americans within BioVU across a range of prevalences

\begin{tabular}{lll}
\hline Heritability estimate \pm SE & $p$ value & Prevalence \\
\hline $0.24 \pm 0.13$ & $0.040^{\mathrm{a}}$ & 0.10 \\
$0.29 \pm 0.16$ & $0.040^{\mathrm{a}}$ & 0.20 \\
$0.33 \pm 0.18$ & $0.040^{\mathrm{a}}$ & 0.30 \\
$0.35 \pm 0.19$ & $0.040^{\mathrm{a}}$ & 0.40 \\
$0.35 \pm 0.20$ & $0.040^{\mathrm{a}}$ & 0.50 \\
$0.35 \pm 0.19$ & $0.040^{\mathrm{a}}$ & 0.60 \\
$0.33 \pm 0.18$ & $0.040^{\mathrm{a}}$ & 0.70 \\
$0.29 \pm 0.16$ & $0.040^{\mathrm{a}}$ & 0.80 \\
$0.24 \pm 0.13$ & $0.040^{\mathrm{a}}$ & 0.90
\end{tabular}

Model: adjusted for age, BMI, and 5 PCs. SNPs are limited to $\geq 5 \%$ MAF. ${ }^{\text {a }}$ Statistically significant. 
Table A3

Heritability estimates for fibroid risk using European Americans within BioVU

\begin{tabular}{lll}
\hline Heritability estimate \pm SE & $p$ value & PC adjustments \\
\hline $0.38 \pm 0.18$ & $0.013^{\mathrm{a}}$ & 0 \\
$0.34 \pm 0.18$ & $0.031^{\mathrm{a}}$ & 1 \\
$0.35 \pm 0.18$ & $0.027^{\mathrm{a}}$ & 2 \\
$0.33 \pm 0.18$ & $0.037^{\mathrm{a}}$ & 3 \\
$0.33 \pm 0.18$ & $0.038^{\mathrm{a}}$ & 4 \\
$0.33 \pm 0.18$ & $0.040^{\mathrm{a}}$ & 5 \\
$0.33 \pm 0.18$ & $0.040^{\mathrm{a}}$ & 6 \\
$0.29 \pm 0.18$ & 0.064 & 7 \\
$0.29 \pm 0.18$ & 0.058 & 8 \\
$0.30 \pm 0.18$ & 0.053 & 9 \\
$0.30 \pm 0.18$ & 0.056 & 10 \\
\hline
\end{tabular}

Model: adjusted for age and BMI and noted PC number in the table. SNPs are limited to $\geq 5 \%$ MAF. Prevalence estimates for fibroids are set at

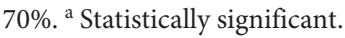

\section{Table A5}

Heritability estimates for the largest fibroid volume using European Americans within BioVU

\begin{tabular}{llc}
\hline Heritability estimate \pm SE & $p$ value & PC adjustments \\
\hline $0.12 \pm 0.65$ & 0.426 & 0 \\
$0.18 \pm 0.65$ & 0.391 & 1 \\
$0.18 \pm 0.65$ & 0.389 & 2 \\
$0.12 \pm 0.66$ & 0.427 & 3 \\
$0.13 \pm 0.66$ & 0.423 & 4 \\
$0.14 \pm 0.66$ & 0.417 & 5 \\
$0.16 \pm 0.65$ & 0.404 & 6 \\
$0.17 \pm 0.67$ & 0.399 & 7 \\
$0.24 \pm 0.67$ & 0.355 & 8 \\
$0.28 \pm 0.67$ & 0.334 & 9 \\
$0.35 \pm 0.67$ & 0.300 & 10 \\
\hline
\end{tabular}

Model: adjusted for age and BMI and noted PC number in the table. SNPs are limited to $\geq 5 \%$ MAF.

\section{Table A4}

Heritability estimates for the largest fibroid dimension using European Americans within BioVU

\begin{tabular}{llc}
\hline Heritability estimate \pm SE & $p$ value & PC adjustments \\
\hline $0.34 \pm 0.47$ & 0.246 & 0 \\
$0.40 \pm 0.47$ & 0.209 & 1 \\
$0.40 \pm 0.47$ & 0.209 & 2 \\
$0.40 \pm 0.47$ & 0.209 & 3 \\
$0.39 \pm 0.47$ & 0.212 & 4 \\
$0.35 \pm 0.47$ & 0.238 & 5 \\
$0.36 \pm 0.48$ & 0.233 & 6 \\
$0.38 \pm 0.48$ & 0.224 & 7 \\
$0.39 \pm 0.48$ & 0.214 & 8 \\
$0.43 \pm 0.48$ & 0.195 & 9 \\
$0.44 \pm 0.48$ & 0.190 & 10
\end{tabular}

Model: adjusted for age and BMI and noted PC number in the table. SNPs are limited to $\geq 5 \%$ MAF.

\section{Table A6}

Heritability estimates for fibroid risk suggestive of significant regions of the genome removed from prior studies

\begin{tabular}{ll}
\hline Studies & Heritability estimate \pm SE \\
\hline No studies & $0.33 \pm 0.18$ \\
1 study & $0.33 \pm 0.18$ \\
2 studies & $0.33 \pm 0.18$ \\
All studies & $0.34 \pm 0.18$
\end{tabular}

Model: adjusted for age, BMI, and 5 PCs. SNPs are limited to $\geq 5 \%$ MAF. Prevalence estimates of fibroids are set at $70 \%$. 
Fig. A1. Principal components plot of BioVU EAs with reference population before pruning outliers. AFR, African; AMR, admixed American; EAS, East Asian; SAS, South Asian; EUR, European.

Fig. A2. Principal components plot of BioVU EAs with reference population after pruning outliers. AFR, African; AMR, admixed American; EAS, East Asian; SAS, South Asian; EUR, European.

Fig. A3. Estimated SNP-based heritability of each chromosome by chromosome length with sections of chromosome 8 removed. To help all models converge, the model used to produce this figure adjusted for age, BMI, and 7 PCs with a prevalence estimate at $70 \%$.
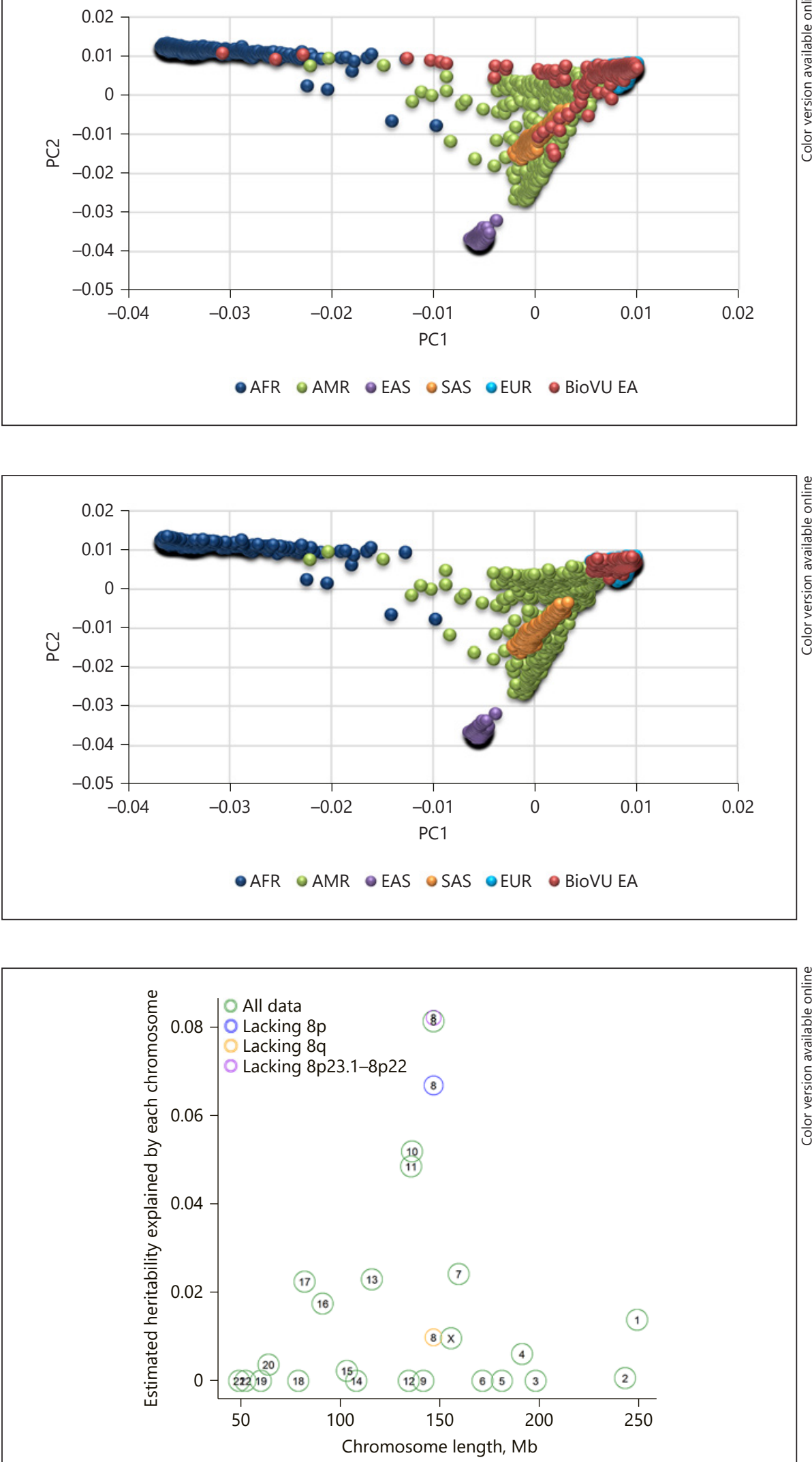


\section{References}

1 Stewart EA. Uterine fibroids. Lancet. 2001 Jan;357(9252):293-8.

2 Baird DD, Dunson DB, Hill MC, Cousins D, Schectman JM. High cumulative incidence of uterine leiomyoma in black and white women: ultrasound evidence. Am J Obstet Gynecol. 2003 Jan;188(1):100-7.

3 De La Cruz MS, Buchanan EM. Uterine Fibroids: diagnosis and Treatment. Am Fam Physician. 2017 Jan;95(2):100-7.

4 Zimmermann A, Bernuit D, Gerlinger C, Schaefers M, Geppert K. Prevalence, symptoms and management of uterine fibroids: an international internet-based survey of 21,746 women. BMC Womens Health. 2012 Mar; 12(1):6.

5 Farquhar CM, Steiner CA. Hysterectomy rates in the United States 1990-1997. Obstet Gynecol. 2002 Feb;99(2):229-34.

6 Cardozo ER, Clark AD, Banks NK, Henne MB, Stegmann BJ, Segars JH: The estimated annual cost of uterine leiomyomata in the United States. Am J Obstet Gynecol. 2012 Mar;206(3):211.e1-9.

7 Snieder H, MacGregor AJ, Spector TD. Genes control the cessation of a woman's reproductive life: a twin study of hysterectomy and age at menopause. J Clin Endocrinol Metab. 1998 Jun;83(6):1875-80.

8 Luoto R, Kaprio J, Rutanen EM, Taipale P, Perola M, Koskenvuo M. Heritability and risk factors of uterine fibroids - the Finnish Twin Cohort study. Maturitas. 2000 Nov;37(1):1526.

9 Ge T, Chen CY, Neale BM, Sabuncu MR, Smoller JW. Phenome-wide heritability analysis of the UK Biobank. PLoS Genet. 2017 Apr;13(4):e1006711.
10 Eggert SL, Huyck KL, Somasundaram P, Kavalla $\mathrm{R}$, Stewart EA, Lu AT, et al. Genomewide linkage and association analyses implicate FASN in predisposition to Uterine Leiomyomata. Am J Hum Genet. 2012 Oct;91(4): 621-8.

11 Cha PC, Takahashi A, Hosono N, Low SK, Kamatani N, Kubo M, et al. A genome-wide association study identifies three loci associated with susceptibility to uterine fibroids. Nat Genet. 2011 May;43(5):447-50.

12 Hellwege JN, Jeff JM, Wise LA, Gallagher CS, Wellons M, Hartmann KE, et al. A multistage genome-wide association study of uterine fibroids in African Americans. Hum Genet. 2017 Oct;136(10):1363-73.

13 Edwards TL, Hartmann KE, Velez Edwards DR. Variants in BET1L and TNRC6B associate with increasing fibroid volume and fibroid type among European Americans. Hum Genet. 2013 Dec;132(12):1361-9.

14 Edwards TL, Michels KA, Hartmann KE, Velez Edwards DR. BET1L and TNRC6B associate with uterine fibroid risk among European Americans. Hum Genet. 2013 Aug; 132(8):943-53.

15 Roden DM, Pulley JM, Basford MA, Bernard GR, Clayton EW, Balser JR, et al. Development of a large-scale de-identified DNA biobank to enable personalized medicine. Clin Pharmacol Ther. 2008 Sep;84(3):362-9.

16 Pulley J, Clayton E, Bernard GR, Roden DM, Masys DR. Principles of human subjects protections applied in an opt-out, de-identified biobank. Clin Transl Sci. 2010 Feb;3(1):42-8.

17 Feingold-Link L, Edwards TL, Jones S, Hartmann KE, Velez Edwards DR. Enhancing uterine fibroid research through utilization of biorepositories linked to electronic medical record data. J Womens Health (Larchmt). 2014 Dec;23(12):1027-32.
18 Purcell S, Neale B, Todd-Brown K, Thomas L, Ferreira MA, Bender D, et al. PLINK: a tool set for whole-genome association and population-based linkage analyses. Am J Hum Genet. 2007 Sep;81(3):559-75.

19 Howie BN, Donnelly P, Marchini J. A flexible and accurate genotype imputation method for the next generation of genome-wide association studies. PLoS Genet. 2009 Jun; 5(6):e1000529.

20 Lee SH, Wray NR, Goddard ME, Visscher PM. Estimating missing heritability for disease from genome-wide association studies. Am J Hum Genet. 2011 Mar;88(3):294-305.

21 Price AL, Patterson NJ, Plenge RM, Weinblatt ME, Shadick NA, Reich D. Principal components analysis corrects for stratification in genome-wide association studies. Nat Genet. 2006 Aug;38(8):904-9.

22 Yang J, Lee SH, Goddard ME, Visscher PM. GCTA: a tool for genome-wide complex trait analysis. Am J Hum Genet. 2011 Jan;88(1): 76-82.

23 Wang K, Li M, Hakonarson H. ANNOVAR: functional annotation of genetic variants from high-throughput sequencing data. $\mathrm{Nu}$ cleic Acids Res. 2010 Sep;38(16):e164.

24 Broman KW, Matsumoto N, Giglio S, Martin CL, Roseberry JA, Zuffardi O, et al. Common long human inversion polymorphism on chromosome. Lecture Notes-Monograph Series; 2003. pp. 237-46.

25 Davis LK, Yu D, Keenan CL, Gamazon ER, Konkashbaev AI, Derks EM, et al. Partitioning the heritability of Tourette syndrome and obsessive compulsive disorder reveals differences in genetic architecture. PLoS Genet. 2013 Oct;9(10):e1003864.

26 Walker CL, Stewart EA. Uterine fibroids: the elephant in the room. Science. 2005 Jun; 308(5728):1589-92. 